\title{
ON THE DIRICHLET TO NEUMANN PROBLEM FOR THE 1-DIMENSIONAL CUBIC NLS EQUATION ON THE HALF-LINE ${ }^{\dagger}$
}

\author{
D. C. ANTONOPOULOU ${ }^{\$}$ AND S. KAMVISSIS*
}

\begin{abstract}
Initial-boundary value problems for 1-dimensional 'completely integrable' equations can be solved via an extension of the inverse scattering method, which is due to Fokas and his collaborators. A crucial feature of this method is that it requires the values of more boundary data than given for a well-posed problem. In the case of cubic NLS, knowledge of the Dirichet data suffices to make the problem well-posed but the Fokas method also requires knowledge of the values of Neumann data. The study of the Dirichlet to Neumann map is thus necessary before the application of the 'Fokas transform'. In this paper, we provide a rigorous study of this map for a large class of decaying Dirichlet data. We show that the Neumann data are also sufficiently decaying and that, hence, the Fokas method can be applied.
\end{abstract}

\section{INTRODUCTION}

In the last twenty years there has been a series of results by Fokas and collaborators on initialboundary value problems for 'completely integrable' equations. Their method (introduced in [10] and further developed in [11], [12], [14, [15], see also the book by Fokas [13] for a comprehensive review) generalises the 'classical' theory of Kruskal et al., cf. for example in [18, which essentially reduces initial value problems to Riemann-Hilbert problems via the scattering transform (and then studies these problems by the method of inverse scattering going back to Gelfand, Levitan and Marchenko). Instead of the usual scattering transform the new transform of Fokas et al. is based on the simultaneous spectral analysis of both the x-problem and the t-problem in the Lax pair. So, initial-boundary value problems are also reduced to Riemann-Hilbert factorisation problems in the complex plane. The Fokas transform theory was rigorously implemented to the NLS equation on the half-line with Schwartz initial and boundary conditions in [15].

Alternatively, initial-boundary value problems for PDEs can be studied via PDE techniques, whose validity extends to non-integrable equations. In this direction, we cite the seminal contributions of Kenig, Ponce, Vega [25], 26] and Bourgain [3] for initial value problems and a (limited and perhaps random) selection of works by Carrol and Bu [4, Bona, Sun and Zhang [2, Colliander and Kenig [5, and Holmer [19] for initial-boundary value problems.

One of the main advantages of the Riemann-Hilbert formulation is that one can use the powerful nonlinear stationary phase and steepest descent theory (see e.g. the book of P. Deift ([6]) and [22]) which gives rigorous results on the asymptotic behavior of solutions to these Riemann-Hilbert

\footnotetext{
${ }^{\dagger}$ Research funded by ARISTEIA II grant no. 3964 from the General Secretariat of Research and Technology, Greece.

* Department of Pure and Applied Mathematics, University of Crete, GR-700 13 Heraklion, Greece, and, Institute of Applied and Computational Mathematics, FORTH, GR-711 10 Heraklion, Greece, email: spyros@tem.uoc.gr.

\$ Department of Mathematics, University of Chester, Thornton Science Park, CH2 4NU, UK, and, Institute of Applied and Computational Mathematics, FORTH, GR-711 10 Heraklion, Greece, email: d.antonopoulou@chester.ac.uk.
} 
problems (as some parameter goes to infinity) and hence it extracts asymptotics for the solution of the associated soliton equation by use of the 'Riemann-Hilbert method'. (See e.g. [9] for the seminal paper on long time asymptotics for mKdV; [21], 23], [24] for the Toda lattice; [8] for KdV; 7] for NLS; see also [20] and [16] for instances of work that makes use of the Fokas theory.)

An important fact about the Fokas method for well-posed initial-boundary value problems, is that it involves unknown boundary values that should be characterised in terms of the given data. The problem of extra boundary values has to be overcome one way or another for the completely rigorous implementation of the Fokas transform method. As far as finite times are concerned, this issue is dealt with by the analysis of a 'global relation' in [15. If we are interested in infinite times however, this has proved to be harder. This is the problem we are solving here, at least in the defocusing case.

In this paper, we consider the non-linear Schrödinger equation (NLS) with cubic non-linearity, posed on the real positive semi-axis $\mathbb{R}^{+}$

$$
\mathrm{i} q_{t}+q_{x x}-2 \lambda|q|^{2} q=0, \quad x>0, \quad 0<t<+\infty,
$$

and initial-boundary data

$$
\begin{aligned}
& q(x, 0)=q_{0}(x), \quad 0 \leq x<+\infty \\
& q(0, t)=Q(t), \quad 0 \leq t<+\infty,
\end{aligned}
$$

where $q_{0}, Q$ are classical functions satisfying the compatibility condition $q_{0}(0)=Q(0)$.

The case $\lambda=1$ is the defocusing case, while $\lambda=-1$ is the focusing case.

Back in 1991, Carrol and $\mathrm{Bu}$ in [4] established the existence of a unique global classical solution $q \in C^{1}\left(L^{2}\right) \cap C^{0}\left(H^{2}\right)$ of the problem (1.1)-(1.2), with $q_{0} \in H^{2}, Q \in C^{2}$ and $q_{0}(0)=Q(0)$, by using PDE theory.

On the other hand, it is well-known 28, that the non-linear Schrödinger equation (NLS) with cubic non-linearity can be written as a Lax pair and that, at least the Cauchy problem is 'completely integrable', in other words it can be solved via the scattering transform.

Furthermore, in [15] the authors used the Fokas transform to solve the problem on the real positive semi-axis, given values for the initial data and Dirichlet data (which make the problem well-posed) and also the Neumann data $P(t):=q_{x}(0, t)$. Actually what is required for that theory to work is that the Neumann data (as well as the Dirichlet data) live in some class with nice decaying properties such that the Fokas scattering transform can be properly defined. This is exactly the problem we consider here: we will provide several reasonably inclusive large classes of Dirichlet data, such that both Dirichlet and Neumann data decay as $t \rightarrow \infty$ fast enough for the scattering method to work.

Obviously, our problem is a special case of the general one treated in [4] and therefore, a global classical solution $q$ uniquely exists. But the Fokas transform method arrives at a Riemann-Hilbert formulation of the problem on the real positive semi-axis, and hence long time asymptotics (as in [15]) or semiclassical asymptotics [20] are also possible.

1.1. Main results and Strategy. In Section 2, we consider $q(0, t), q_{t}(0, t)$ with mild polynomial decay as $t \rightarrow \infty$ and prove that the Neumann data $q_{x}(0, t)$ is bounded in the $L^{2}(0, \infty)$-norm, while $q(x, t)$ is bounded in the $H^{1}(0, \infty)$-norm in space uniformly for any $t \geq 0$; these arguments are established for any real $\lambda$.

The defocusing NLS is further analyzed in Section 3. We first demonstrate that the NLS solution decays to zero for large times. This yields sharper estimates for the norm $\|q(\cdot, t)\|_{H^{1}(0, \infty)}$ which are crucial for the proof of the main theorem of this Section, which states that if the Dirichlet data 
$q(0, t), q_{t}(0, t)$ and $q_{t t}(0, t)$ have sufficient polynomial decay then $q_{x}(0, t)$ also have good decay and thus the Fokas method is applicable.

For the focusing NLS, analyzed in Section 4, the same result is derived under the assumption of decay of the solution as $t \rightarrow \infty$, as well as a smallness assumption for $\int_{0}^{\infty}|q(0, t)|^{2} d t$. Finally, in Section 5 we present our concluding remarks.

Our strategy for attacking the problem consists of the following steps:

(1) In Theorem 2.1 we estimate

$$
\int_{0}^{\infty}\left|q_{x}(0, t)\right|^{2} d t
$$

in terms of the Dirichlet data.

(2) We prove that if these data decay polynomially, then the above norm is bounded, while $q$ is bounded in $H^{1}(0, \infty)$-norm in space, uniformly for any $t \geq 0$.

(3) For the defocusing NLS, we establish a zero limiting profile of the solution for large times, cf. Theorem 3.1.

(4) We then proceed (using this decay) to prove sharper estimates for $\|q(\cdot, t)\|_{H^{1}(0, \infty)}$ in Theorem 3.4. This results in an estimate of

$$
\mathcal{A}:=\int_{t}^{\infty}\left|q_{x}(0, r)\right|^{2} d r
$$

in terms of the Dirichlet data. In the proof of Theorem 3.6. we provides a bound for

$$
\mathcal{B}:=\int_{0}^{\infty}\left|q_{x t}(0, t)\right|^{2} d t
$$

(5) Finally, we estimate $\left|q_{x}(0, t)\right|$ by using the bounds of $\mathcal{A}$ and $\mathcal{B}$, to obtain the paper's main result, which is Theorem 3.8 .

(6) Considering the focusing NLS, the strategy is similar, but here we need the assumption that the solution decays at large times, a fact that we are able to prove only for the defocusing case.

\section{General estimates}

In this section, we prove some basic inequalities for the NLS solution, for any real non-zero $\lambda$. Hence, these results are applicable for both the defocusing and focusing case.

In what follows $(\cdot, \cdot)$ will denote the $L^{2}(0, \infty)$ inner product in space variables, while $\|\cdot\|$ the induced norm. Furthermore, we shall use the symbol $\|\cdot\|_{4}$ for the $L^{4}(0, \infty)$ norm in space and for any integer $p \geq 1,\|\cdot\|_{L^{p}(0, t)}$ will denote the $L^{p}$ norm in the time interval $(0, t)$.

The symbol $c$ will be used in general to denote nonnegative constants.

Throughout this paper we make heavy use of the decay of the solution $q$ of the NLS problem (1.1)- (1.2)

$$
q(x, t) \rightarrow 0 \text { as } \quad x \rightarrow \infty \text { for any } t \geq 0,
$$

as of course is guaranteed by the existence of a unique classical solution in [4]. For simplicity, we also assume here that

$$
q(x, 0)=q_{0}(x)=0 \quad \text { for any } x \geq 0 .
$$

This simplifying assumption will be dropped in a forthcoming addendum to this paper.

The next Theorem presents a first general estimate in $L^{2}(0, t)$-norm of the Neumann data $q_{x}(0, \cdot)$ valid for $t \rightarrow \infty$ also. In fact, in order to obtain a uniform estimate for any $t \geq 0$, we extent the 
proof of [15], cf. Appendix D1, which was only established for $t$ taking values in a bounded domain, thus involving constants that may depend on its upper bound.

Theorem 2.1. Let $q$ be the unique global classical solution $q \in C^{1}\left(L^{2}\right) \cap C^{0}\left(H^{2}\right)$ of the problem (1.1) -(1.2), with $Q \in C^{2}$ and $Q(0)=0$, under the assumption (2.2). It holds that for any $t \geq 0$

$$
\left(\int_{0}^{t}\left|q_{x}(0, s)\right|^{2} d s\right)^{1 / 2}=\left\|q_{x}(0, \cdot)\right\|_{L^{2}(0, t)} \leq c\|q(0, \cdot)\|_{L^{2}(0, t)}^{1 / 2}\left\|q_{t}(0, \cdot)\right\|_{L^{2}(0, t)}^{1 / 2}+c_{1}\|q(0, \cdot)\|_{L^{4}(0, t)}^{2},
$$

for c positive constant independent of $t, c_{1} \geq 0$ constant independent of $t$ with $c_{1}=0$ if $\lambda<0$ and $c_{1}>0$ if $\lambda>0$.

For the case $\lambda<0$, we also impose the additional assumption that $\|q(0, \cdot)\|_{L^{2}(0, t)}^{2} \leq c_{2}$ uniformly in $t \geq 0$, for some $c_{2}>0$ sufficiently small.

The inequality (2.3) is also true when $t$ is replaced by $\infty$.

Remark 2.2. We observe that in Appendix D1 of [15] relation (D.10) is proven for bounded time intervals $(0, T)$ and involving a general continuous map of the $H^{1}(0, T)$ norm of $q(0, \cdot)=Q(t)$. In this theorem we shall include the case $t \rightarrow \infty$, while in view of (2.3), we specify this map in terms of specific powers of the appearing norms.

Proof. Multiplying (1.1) by $\bar{q}$ and integrating in $x \in(0, \infty)$ we obtain after applying integration by parts

$$
\mathrm{i}\left(q_{t}, q\right)-\left\|q_{x}\right\|^{2}-q_{x}(0, t) \bar{q}(0, t)-2 \lambda\left(|q|^{2} q, q\right)=0 .
$$

Taking imaginary parts we arrive at

$$
\frac{d}{d t}\|q\|^{2}=2 \operatorname{Im}\left\{q_{x}(0, t) \bar{q}(0, t)\right\}
$$

which after integration in time gives

$$
\|q(\cdot, t)\|^{2} \leq 2\left\|q_{x}(0, \cdot)\right\|_{L^{2}(0, t)}\|q(0, \cdot)\|_{L^{2}(0, t)} .
$$

Multiplying now (1.1) by $\bar{q}_{t}$ and integrating in $x \in(0, \infty)$ we obtain

$$
\mathrm{i}\left\|q_{t}\right\|^{2}-\left(q_{x}, q_{x t}\right)-q_{x}(0, t) \bar{q}_{t}(0, t)-2 \lambda\left(|q|^{2} q, q_{t}\right)=0,
$$

while taking real parts and since

$$
\frac{d}{d t}\left[|q|^{2}|q|^{2}\right]=4 \operatorname{Re}\left\{|q|^{2} q \bar{q}_{t}\right\}
$$

we have

$$
\frac{d}{d t}\left\|q_{x}\right\|^{2}=-\lambda \frac{d}{d t}\left\||q|^{2}\right\|^{2}-2 \operatorname{Re}\left\{q_{x}(0, t) \bar{q}_{t}(0, t)\right\} .
$$

Integration in time yields

$$
\left\|q_{x}(\cdot, t)\right\|^{2}=-\lambda\left\||q(\cdot, t)|^{2}\right\|^{2}-2 \int_{0}^{t} \operatorname{Re}\left\{q_{x}(0, s) \bar{q}_{t}(0, s)\right\} d s
$$

and thus,

$$
\left\|q_{x}(\cdot, t)\right\|^{2}+\lambda\|q(\cdot, t)\|_{L^{4}(0, \infty)}^{4} \leq 2\left\|q_{x}(0, \cdot)\right\|_{L^{2}(0, t)}\left\|q_{t}(0, \cdot)\right\|_{L^{2}(0, t)} .
$$

We now multiply (1.1) with $\overline{q_{x}}$ integrate in space and take real parts which yields

$$
-\operatorname{Im}\left(q_{t}, q_{x}\right)+\operatorname{Re}\left(q_{x x}, q_{x}\right)-2 \lambda \operatorname{Re}\left(|q|^{2} q, q_{x}\right)=0 .
$$


Using that

and the relations

$$
\operatorname{Im}\left(q_{t}, q_{x}\right)=-\frac{1}{2} \mathrm{i} \frac{d}{d t}\left(q, q_{x}\right)-\frac{1}{2} \mathrm{i} q(0, t) \bar{q}_{t}(0, t)
$$

$$
\operatorname{Re}\left(q_{x x}, q_{x}\right)=-\frac{1}{2}\left|q_{x}(0, t)\right|^{2}, \quad \operatorname{Re}\left(|q|^{2} q, q_{x}\right)=-\frac{1}{4}|q(0, t)|^{4},
$$

we obtain

$$
\left|q_{x}(0, t)\right|^{2}=\mathrm{i} \frac{d}{d t}\left(q, q_{x}\right)+\mathrm{i} q(0, t) \bar{q}_{t}(0, t)+\lambda|q(0, t)|^{4} .
$$

Integrating the above in time, we get

$$
\int_{0}^{t}\left|q_{x}(0, s)\right|^{2} d s=\mathrm{i}\left(q(\cdot, t), q_{x}(\cdot, t)\right)+\mathrm{i} \int_{0}^{t} q(0, s) \bar{q}_{t}(0, s) d s+\lambda \int_{0}^{t}|q(0, s)|^{4} d s,
$$

and so,

$$
\left\|q_{x}(0, \cdot)\right\|_{L^{2}(0, t)}^{2} \leq\|q(\cdot, t)\|\left\|q_{x}(\cdot, t)\right\|+\|q(0, \cdot)\|_{L^{2}(0, t)}\left\|q_{t}(0, \cdot)\right\|_{L^{2}(0, t)}+\lambda\|q(0, \cdot)\|_{L^{4}(0, t)}^{4} .
$$

We shall estimate $\|q(\cdot, t)\|\left\|q_{x}(\cdot, t)\right\|$. Indeed, by (2.5) and (2.7) and the Sobolev inequality

$$
\|q(\cdot, t)\|_{L^{4}(0, \infty)}^{4} \leq\|q(\cdot, t)\|^{3}\left\|q_{x}(\cdot, t)\right\|
$$

we arrive at

$$
\begin{aligned}
\|q(\cdot, t)\|\left\|q_{x}(\cdot, t)\right\| \leq & c\left\|q_{x}(0, \cdot)\right\|_{L^{2}(0, t)}\|q(0, \cdot)\|_{L^{2}(0, t)}^{1 / 2}\left\|q_{t}(0, \cdot)\right\|_{L^{2}(0, t)}^{1 / 2} \\
& +\tilde{c}\left\|q_{x}(0, \cdot)\right\|_{L^{2}(0, t)}^{1 / 2}\|q(0, \cdot)\|_{L^{2}(0, t)}^{1 / 2}\|q(\cdot, t)\|^{3 / 2}\left\|q_{x}(\cdot, t)\right\|^{1 / 2} \\
\leq & c\left\|q_{x}(0, \cdot)\right\|_{L^{2}(0, t)}\|q(0, \cdot)\|_{L^{2}(0, t)}^{1 / 2}\left\|q_{t}(0, \cdot)\right\|_{L^{2}(0, t)}^{1 / 2} \\
& +\tilde{c} c\left\|q_{x}(0, \cdot)\right\|_{L^{2}(0, t)}\|q(0, \cdot)\|_{L^{2}(0, t)}\|q(\cdot, t)\|^{2}+\tilde{c} c_{0}\|q(\cdot, t)\|\left\|q_{x}(\cdot, t)\right\|,
\end{aligned}
$$

where $\tilde{c}=0$ if $\lambda=1$ and $c_{0}$ can be made as small as we want. Hence, using the above and the estimate (2.5) for $\|q\|$, we have

$$
\begin{aligned}
\|q(\cdot, t)\|\left\|q_{x}(\cdot, t)\right\| \leq & c\left\|q_{x}(0, \cdot)\right\|_{L^{2}(0, t)}\|q(0, \cdot)\|_{L^{2}(0, t)}^{1 / 2}\left\|q_{t}(0, \cdot)\right\|_{L^{2}(0, t)}^{1 / 2} \\
& +\tilde{c} c\left\|q_{x}(0, \cdot)\right\|_{L^{2}(0, t)}\|q(0, \cdot)\|_{L^{2}(0, t)}\|q(\cdot, t)\|^{2} \\
\leq & c\left\|q_{x}(0, \cdot)\right\|_{L^{2}(0, t)}\|q(0, \cdot)\|_{L^{2}(0, t)}^{1 / 2}\left\|q_{t}(0, \cdot)\right\|_{L^{2}(0, t)}^{1 / 2}+\tilde{c} c\left\|q_{x}(0, \cdot)\right\|_{L^{2}(0, t)}^{2}\|q(0, \cdot)\|_{L^{2}(0, t)}^{2} \\
\leq & \hat{c}_{0}\left\|q_{x}(0, \cdot)\right\|_{L^{2}(0, t)}^{2}+c\|q(0, \cdot)\|_{L^{2}(0, t)}\left\|q_{t}(0, \cdot)\right\|_{L^{2}(0, t)} \\
& +\tilde{c} c\left\|q_{x}(0, \cdot)\right\|_{L^{2}(0, t)}^{2}\|q(0, \cdot)\|_{L^{2}(0, t)}^{2},
\end{aligned}
$$

where again $\hat{c}_{0}$ can be made as small as we want. Using now (2.10) in (2.9) we obtain

$$
\begin{aligned}
\left\|q_{x}(0, \cdot)\right\|_{L^{2}(0, t)}^{2} \leq & \hat{c}_{0}\left\|q_{x}(0, \cdot)\right\|_{L^{2}(0, t)}^{2}+c\|q(0, \cdot)\|_{L^{2}(0, t)}\left\|q_{t}(0, \cdot)\right\|_{L^{2}(0, t)} \\
& +\tilde{c} c\left\|q_{x}(0, \cdot)\right\|_{L^{2}(0, t)}^{2}\|q(0, \cdot)\|_{L^{2}(0, t)}^{2}+\|q(0, \cdot)\|_{L^{2}(0, t)}\left\|q_{t}(0, \cdot)\right\|_{L^{2}(0, t)}+\lambda\|q(0, \cdot)\|_{L^{4}(0, t)}^{4},
\end{aligned}
$$

and thus we obtain (2.3), i.e.

$$
\left\|q_{x}(0, \cdot)\right\|_{L^{2}(0, t)}^{2} \leq c\|q(0, \cdot)\|_{L^{2}(0, t)}\left\|q_{t}(0, \cdot)\right\|_{L^{2}(0, t)}+c_{1}\|q(0, \cdot)\|_{L^{4}(0, t)}^{4},
$$


where $c_{1}=c_{1}(\lambda)=0$ if $\lambda=-1$, because we had made $\hat{c}_{0}$ appropriately small; when $\lambda=-1$, i.e. when $\tilde{c} \neq 0$ we use the assumption that $\|q(0, \cdot)\|_{L^{2}(0, t)}^{2}$ is small uniformly in $t$.

Remark 2.3. Under the assumptions of the previous Theorem [2.1] and due to estimate (2.3), we note that if $q(0, t), q_{t}(0, t)$, have polynomial decay of order $\mathcal{O}\left(t^{-\alpha}\right), \mathcal{O}\left(t^{-\beta}\right)$ respectively as $t \rightarrow \infty$, with $\alpha, \beta>1 / 2$ then:

1) It holds that

$$
\int_{0}^{\infty}\left|q_{x}(0, t)\right|^{2} d t<\infty
$$

2) Furthermore for $\alpha, \beta$ as in 1), the estimate (2.11), together with (2.5) and (2.7) yield that there exists $c>0$ independent of $t$, such that for any $t \geq 0$

$$
\|q(\cdot, t)\| \leq c
$$

and

$$
\left\|q_{x}(\cdot, t)\right\| \leq c .
$$

(Note that in order to prove the inequality (2.13), and since an $L^{4}$-norm term is present in (2.7), we use additionally the Sobolev Embedding Theorem

$$
\|q\|_{4}^{4} \leq\|q\|^{3}\left\|q_{x}\right\| \leq c\|q\|^{6}+c_{0}\left\|q_{x}\right\|^{2},
$$

where $c_{0}>0$ can be made as small as we want.)

For $\alpha, \beta$ sufficiently large, we shall eventually prove sharper estimates for these norms.

3) In addition, we have that there exists $c>0$ independent of $t$ such that for any $t \geq 0$

$$
|q(x, t)| \leq c\|q(\cdot, t)\|^{1 / 2}\left\|q_{x}(\cdot, t)\right\|^{1 / 2} \leq c,
$$

and so,

$$
\lim _{t \rightarrow \infty}|q(x, t)| \leq c,
$$

for any $x$, if the limit exists.

\section{Defocusing NLS}

We now proceed by considering the case $\lambda=1$, i.e. the defocusing NLS equation.

Our first aim is to establish, under certain assumptions for the initial data, that the solution of (1.1)-(1.2) when $\lambda=1$ decays to 0 for any $x$ as $t \rightarrow \infty$. This is achieved by proving that the $L^{4}$ norm of the solution decays in time.

More specifically, we prove the next main theorem. 
Theorem 3.1. Let $q$ be the unique global classical solution $q \in C^{1}\left(L^{2}\right) \cap C^{0}\left(H^{2}\right)$ of the problem (1.1)-(1.2), with $Q \in C^{2}$ and $Q(0)=0$, with $\lambda=1$, under the assumption (2.2). Furthermore, assume that as $t \rightarrow \infty$

$$
q(0, t)=\mathcal{O}\left(t^{-\alpha}\right), \quad q_{t}(0, t)=\mathcal{O}\left(t^{-\beta}\right), \quad \text { for } \alpha>3 / 2 \quad \text { and } \beta>5 / 2 .
$$

It holds that there exists a positive constant $c$ independent of $t$ such that

$$
\int_{0}^{\infty}|q(x, t)|^{4} d x:=\|q(\cdot, t)\|_{4}^{4} \leq \frac{c}{t} \quad \text { for any } t \geq 1 .
$$

Proof. Again let the Dirichlet data be

$$
Q(t):=q(0, t)
$$

and let us denote the Neumann data by

$$
P(t):=q_{x}(0, t) .
$$

We set

$$
A(t):=\int_{0}^{\infty}\left(\left|q_{x}(x, t)\right|^{2}+|q(x, t)|^{4}\right) d x
$$

Then by (2.6) we have

$$
\partial_{t} A(t)=-2 \operatorname{Re}\left\{q_{x}(0, t) \bar{q}_{t}(0, t)\right\}=-2 \operatorname{Re}\{P(t) \bar{Q}(t)\} .
$$

We now define

$$
y(t)=\operatorname{Im} \int_{0}^{\infty} x \bar{q}(x, t) q_{x}(x, t) d x
$$

to obtain

$$
\begin{aligned}
y_{t}=\operatorname{Im} \int_{0}^{\infty}\left(x \overline{q_{t}} q_{x}+x \bar{q} q_{x t}\right) d x & =\operatorname{Im} \int_{0}^{\infty}\left(x \overline{q_{t}} q_{x}-\bar{q} q_{t}-x \overline{q_{x}} q_{t}\right) d x+\operatorname{Im}\left[x \bar{q} q_{t}\right]_{x=0}^{\infty} \\
& =-\operatorname{Im} \int_{0}^{\infty} \bar{q} q_{t} d x+2 \operatorname{Im} \int_{0}^{\infty} x \overline{q_{t}} q_{x} d x
\end{aligned}
$$

Using the NLS equation (1.1), we have

$$
\mathrm{i}\left(x q_{t}, q_{x}\right)+\left(x q_{x x}, q_{x}\right)-2\left(x|q|^{2} q, q_{x}\right)=0,
$$

which gives, by taking real parts,

$$
-\operatorname{Im}\left(x q_{t}, q_{x}\right)+\operatorname{Re}\left(x q_{x x}, q_{x}\right)-2 \operatorname{Re}\left(x|q|^{2} q, q_{x}\right)=0 .
$$

We observe that

$$
\left(x q_{x x}, q_{x}\right)=-\left(q_{x}, q_{x}\right)-\left(x q_{x}, q_{x x}\right)+\left[x\left|q_{x}\right|^{2}\right]_{x=0}^{\infty},
$$

and thus,

$$
\operatorname{Re}\left(x q_{x x}, q_{x}\right)=-\frac{1}{2}\left\|q_{x}\right\|^{2}
$$

Furthermore,

so, we obtain

$$
\left(x|q|^{2} q, q_{x}\right)=-\int_{0}^{\infty}|q|^{4} d x-\int_{0}^{\infty} x\left[2 q_{x}|q|^{2} \bar{q}+q \overline{q_{x}}|q|^{2}\right] d x
$$

$$
\operatorname{Re}\left(x|q|^{2} q, q_{x}\right)=-\frac{1}{4} \int_{0}^{\infty}|q|^{4} d x
$$


Relations (3.4), (3.5), (3.6), yield

$$
0=-\operatorname{Im}\left(x q_{t}, q_{x}\right)-\frac{1}{2}\left\|q_{x}\right\|^{2}+\frac{1}{2} \int_{0}^{\infty}|q|^{4} d x .
$$

In addition, the NLS equation (1.1) gives

$$
\mathrm{i}\left(q_{t}, q\right)+\left(q_{x x}, q\right)-2\left(|q|^{2} q, q\right)=0,
$$

thus, taking real parts, we obtain

$$
-\operatorname{Im}\left(q_{t}, q\right)+\operatorname{Re}\left(q_{x x}, q\right)-2 \operatorname{Re}\left(|q|^{2} q, q\right)=0,
$$

while

$$
\left(q_{x x}, q\right)=-\left\|q_{x}\right\|^{2}+\left[q_{x} \bar{q}\right]_{x=0}^{\infty}=-\left\|q_{x}\right\|^{2}-q_{x}(0, t) \bar{q}(0, t) .
$$

By (3.8), (3.9) we have

$$
0=-\operatorname{Im}\left(q_{t}, q\right)-\left\|q_{x}\right\|^{2}-\operatorname{Re}\left\{q_{x}(0, t) \bar{q}(0, t)\right\}-2 \int_{0}^{\infty}|q|^{4} d x .
$$

Therefore, (3.3), together with (3.10), and (3.7) give

$$
\begin{aligned}
y_{t}(t) & =2\left\|q_{x}\right\|^{2}+\operatorname{Re}\left\{q_{x}(0, t) \bar{q}(0, t)\right\}+\int_{0}^{\infty}|q|^{4} d x \\
& =\operatorname{Re} P(t) \bar{Q}(t)+A(t)+\int_{0}^{\infty}\left|q_{x}(x, t)\right|^{2} d x .
\end{aligned}
$$

Observe now that

$$
\partial_{t} \int_{0}^{\infty} x^{2}|q|^{2} d x=\int_{0}^{\infty} x^{2}\left[q_{t} \bar{q}+q \bar{q} t\right] d x=2 \operatorname{Re} \int_{0}^{\infty} x^{2} q_{t} \bar{q} d x .
$$

Multiplying the NLS equation (1.1) by $x^{2}$ we obtain

$$
\begin{aligned}
0 & =\mathrm{i}\left(x^{2} q_{t}, q\right)+\left(x^{2} q_{x x}, q\right)-2\left(x^{2}|q|^{2} q, q\right) \\
& =\mathrm{i}\left(x^{2} q_{t}, q\right)-\left(2 x q_{x}, q\right)-\left(x^{2} q_{x}, q_{x}\right)+\left[x^{2} q_{x} \bar{q}\right]_{x=0}^{\infty}-2 \int_{0}^{\infty} x^{2}|q|^{4} d x \\
& =\mathrm{i}\left(x^{2} q_{t}, q\right)-\left(2 x q_{x}, q\right)-\left(x^{2} q_{x}, q_{x}\right)-2 \int_{0}^{\infty} x^{2}|q|^{4} d x .
\end{aligned}
$$

So, taking imaginary parts yields

$$
2 \operatorname{Re}\left(x^{2} q_{t}, q\right)=2 \operatorname{Im}\left(2 x q_{x}, q\right)=4 y,
$$

which gives by (3.12)

$$
\partial_{t} \int_{0}^{\infty} x^{2}|q|^{2} d x=4 y
$$

But, we have

$$
4 t y_{t}=\partial_{t}(4 t y)-4 y
$$

and thus, by (3.11) and (3.13), we obtain

$$
4 t \operatorname{Re}\{P \bar{Q}\}+4 t A+4 t \int_{0}^{\infty}\left|q_{x}\right|^{2} d x=\partial_{t}(4 t y)-\partial_{t} \int_{0}^{\infty} x^{2}|q|^{2} d x,
$$


and equivalently, replacing $A$

$$
\begin{aligned}
\partial_{t}(4 t y) & =4 t \operatorname{Re}\{P \bar{Q}\}+4 t A+4 t \int_{0}^{\infty}\left|q_{x}\right|^{2} d x+\partial_{t} \int_{0}^{\infty} x^{2}|q|^{2} d x \\
& =4 t \operatorname{Re}\{P \bar{Q}\}+8 t \int_{0}^{\infty}\left|q_{x}\right|^{2} d x+4 t \int_{0}^{\infty}|q|^{4} d x+\partial_{t} \int_{0}^{\infty} x^{2}|q|^{2} d x \\
& =4 t \operatorname{Re}\{P \bar{Q}\}+\partial_{t}\left(4 t^{2} \int_{0}^{\infty}\left|q_{x}\right|^{2} d x\right)-4 t^{2} \int_{0}^{\infty} \partial_{t}\left|q_{x}\right|^{2} d x+4 t \int_{0}^{\infty}|q|^{4} d x+\partial_{t} \int_{0}^{\infty} x^{2}|q|^{2} d x
\end{aligned}
$$

So, using (3.14) we arrive at

$$
\begin{aligned}
& \partial_{t}\left[4 t y-\int_{0}^{\infty} x^{2}|q|^{2} d x-4 t^{2} \int_{0}^{\infty}\left|q_{x}\right|^{2} d x\right]= \\
& 4 t \operatorname{Re}\{P \bar{Q}\}-4 t^{2} \int_{0}^{\infty} \partial_{t}\left|q_{x}\right|^{2} d x+4 t \int_{0}^{\infty}|q|^{4} d x .
\end{aligned}
$$

Using (2.6) in (3.15), we obtain

$$
\begin{aligned}
& \partial_{t}\left[4 t y-\int_{0}^{\infty} x^{2}|q|^{2} d x-4 t^{2} \int_{0}^{\infty}\left|q_{x}\right|^{2} d x\right]= \\
& 4 t \operatorname{Re}\{P \bar{Q}\}+4 t^{2} \partial_{t}\left(\int_{0}^{\infty}|q|^{4} d x\right)+8 t^{2} \operatorname{Re}\left\{P \bar{Q}_{t}\right\}+4 t \int_{0}^{\infty}|q|^{4} d x .
\end{aligned}
$$

But,

$$
4 t^{2} \partial_{t}\left(\int_{0}^{\infty}|q|^{4} d x\right)+4 t \int_{0}^{\infty}|q|^{4} d x=\partial_{t}\left(4 t^{2} \int_{0}^{\infty}|q|^{4} d x\right)-4 t \int_{0}^{\infty}|q|^{4} d x
$$

and therefore, (3.16) yields

$$
\begin{aligned}
& \partial_{t}\left[4 t y-\int_{0}^{\infty} x^{2}|q|^{2} d x-4 t^{2} \int_{0}^{\infty}\left|q_{x}\right|^{2} d x-4 t^{2} \int_{0}^{\infty}|q|^{4} d x\right] \\
& =4 t \operatorname{Re}\{P \bar{Q}\}+8 t^{2} \operatorname{Re}\left\{P \bar{Q}_{t}\right\}-4 t \int_{0}^{\infty}|q|^{4} d x
\end{aligned}
$$

and so,

$$
\begin{aligned}
\partial_{t}\left(t^{2} \int_{0}^{\infty}|q|^{4} d x\right)= & \frac{1}{4} \partial_{t}\left[4 t y-\int_{0}^{\infty} x^{2}|q|^{2} d x-4 t^{2} \int_{0}^{\infty}\left|q_{x}\right|^{2} d x\right] \\
& -t \operatorname{Re}\{P \bar{Q}\}-2 t^{2} \operatorname{Re}\left\{P \bar{Q}_{t}\right\}+t \int_{0}^{\infty}|q|^{4} d x
\end{aligned}
$$

Integrating the above in time in the interval $(0, t)$, we get

$$
\begin{aligned}
t^{2}\|q(\cdot, t)\|_{4}^{4}= & \frac{1}{4}\left[4 t y-\int_{0}^{\infty} x^{2}|q(x, t)|^{2} d x-4 t^{2} \int_{0}^{\infty}\left|q_{x}(x, t)\right|^{2} d x\right] \\
& -\int_{0}^{t} r \operatorname{Re}\{P(r) \bar{Q}(r)\} d r-2 \int_{0}^{t} r^{2} \operatorname{Re}\left\{P(r) \bar{Q}_{r}(r)\right\} d r+\int_{0}^{t} r\|q(\cdot, r)\|_{4}^{4} d r .
\end{aligned}
$$

Observe now that

$$
4 t y=\int_{0}^{\infty}\left[x^{2}|q|^{2}+4 t^{2}\left|q_{x}\right|^{2}-\left|x q+2 \mathrm{i} t q_{x}\right|^{2}\right] d x
$$


While by (3.17) we have

$$
\begin{aligned}
t^{2}\|q(\cdot, t)\|_{4}^{4}=\frac{1}{4}[ & \int_{0}^{\infty}\left(x^{2}|q(x, t)|^{2}+4 t^{2}\left|q_{x}(x, t)\right|^{2}-\left|x q+2 \mathrm{i} t q_{x}(x, t)\right|^{2}-x^{2}|q(x, t)|^{2}\right) d x \\
& \left.-4 t^{2} \int_{0}^{\infty}\left|q_{x}(x, t)\right|^{2} d x\right] \\
& -\int_{0}^{t} r \operatorname{Re}\{P(r) \bar{Q}(r)\} d r-2 \int_{0}^{t} r^{2} \operatorname{Re}\left\{P(r) \bar{Q}_{r}(r)\right\} d r+\int_{0}^{t} r\|q(\cdot, r)\|_{4}^{4} d r \\
= & \frac{1}{4}\left[-\int_{0}^{\infty}\left|x q(x, t)+2 \mathrm{i} t q_{x}(x, t)\right|^{2} d x\right] \\
& -\int_{0}^{t} r \operatorname{Re}\{P(r) \bar{Q}(r)\} d r-2 \int_{0}^{t} r^{2} \operatorname{Re}\left\{P(r) \bar{Q}_{r}(r)\right\} d r+\int_{0}^{t} r \|\left. q(\cdot, r)\right|_{4} ^{4} d r .
\end{aligned}
$$

Hence, (3.19) gives

$$
t^{2}\|q(\cdot, t)\|_{4}^{4} \leq \int_{0}^{t} r\|q(\cdot, r)\|_{4}^{4} d r+F\left(P, Q, Q_{t}, t\right)
$$

for

$$
F\left(P, Q, Q_{t}, t\right):=-\int_{0}^{t} r \operatorname{Re}\{P(r) \bar{Q}(r)\} d r-2 \int_{0}^{t} r^{2} \operatorname{Re}\left\{P(r) \bar{Q}_{r}(r)\right\} d r .
$$

Next, we prove that there exists $c>0$ independent of $t$ such that for any $t \geq 0$,

$$
\left|F\left(P, Q, Q_{t}, t\right)\right| \leq c
$$

Indeed, if $a>3 / 2$ and $\beta>5 / 2$, then we have for $F=F\left(P(t), Q(t), Q_{t}(t), t\right)$

$$
\begin{aligned}
|F| & \leq c\left(\int_{0}^{t}|P(r)|^{2} d r\right)^{1 / 2}\left(\int_{0}^{t} r^{2}|Q(r)|^{2} d r\right)^{1 / 2}+c\left(\int_{0}^{t}|P(r)|^{2} d r\right)^{1 / 2}\left(\int_{0}^{t} r^{4}\left|Q_{r}(r)\right|^{2} d r\right)^{1 / 2} \\
& \leq c\left(\int_{0}^{t}|P(r)|^{2} d r\right)^{1 / 2} \leq c
\end{aligned}
$$

since (2.11) is true. Thus, (3.21) follows.

Using now (3.21) at (3.20), we obtain that there exists $c>0$ independent of $t$ such that

$$
t^{2}\|q(\cdot, t)\|_{4}^{4} \leq \int_{0}^{t} r\|q(\cdot, r)\|_{4}^{4} d r+c,
$$

and therefore, using (2.7) for $\lambda>0$, we have for any $t \geq 1$

$$
\begin{aligned}
t^{2}\|q(\cdot, t)\|_{4}^{4} & \leq \int_{0}^{1} r\|q(\cdot, r)\|_{4}^{4} d r+\int_{1}^{t} r\|q(\cdot, r)\|_{4}^{4} d r+c \\
& \leq \int_{0}^{1}\|q(\cdot, r)\|_{4}^{4} d r+\int_{1}^{t} r\|q(\cdot, r)\|_{4}^{4} d r+c \\
& \leq c\left(\int_{0}^{\infty}\left|q_{x}(0, r)\right|^{2} d r\right)^{1 / 2}\left(\int_{0}^{\infty}\left|q_{t}(0, r)\right|^{2} d r\right)^{1 / 2}+\int_{1}^{t} r\|q(\cdot, r)\|_{4}^{4} d r+c \\
& \leq \int_{1}^{t} r\|q(\cdot, r)\|_{4}^{4} d r+c
\end{aligned}
$$


where we used (2.11) and the fact that $\beta>1 / 2$. So, there exists $c>0$ independent of $t$ such that for any $t \geq 1$

$$
t^{2}\|q(\cdot, t)\|_{4}^{4} \leq \int_{1}^{t} r\|q(\cdot, r)\|_{4}^{4} d r+c .
$$

Applying Gronwall's lemma to the above, we obtain (3.1).

Remark 3.2. Similar relations to (3.1) have been proved in [1, 4] for the defocusing NLS; in [1] for zero Dirichlet data $q(0, t)$, while in [4] under additional assumptions.

But here we have provided the first sufficient polynomial decay conditions as $t \rightarrow \infty$ involving not only $q(0, t)=Q(t)$ but $q_{t}(0, t)=Q_{t}(t)$ as well, which make the $L^{4}$-norm decay true. This permits us to establish a zero limiting behaviour for the solution, uniformly in space, as $t \rightarrow \infty$, which is crucial for proving the main results of this paper, i.e. Theorems 3.8 and 4.4.

A direct corollary of the previous theorem is presented in the next proposition.

Proposition 3.3. Under the assumptions of Theorem 3.1 it holds that the pointwise limits as $t \rightarrow \infty$ are

$$
\lim _{t \rightarrow \infty} q(x, t)=0, \quad \lim _{t \rightarrow \infty}\left(\partial_{x}^{k} q\right)(x, t)=0, \quad \lim _{t \rightarrow \infty} q_{t}(x, t)=0, \quad \lim _{t \rightarrow \infty}\left(\partial_{x}^{k} \partial_{t} q\right)(x, t)=0,
$$

for any $x>0$, and any integer $k \geq 1$.

Proof. Indeed, we have by Theorem 3.1

$$
\lim _{t \rightarrow \infty}\|q(\cdot, t)\|_{4} \leq \lim _{t \rightarrow \infty} \frac{c}{t^{1 / 4}}=0
$$

which implies the first two relations of (3.23). In fact, observing that

$$
\frac{d}{d x} q^{3}(x, t)=3 q^{2}(x, t) q_{x}(x, t)
$$

we have

$$
\begin{aligned}
\left|q^{3}(x, t)\right| & =\left|q^{3}(0, t)+\int_{0}^{x} 3 q^{2}(y, t) q_{x}(y, t) d y\right| \\
& \leq|q(0, t)|^{3}+c\left(\int_{0}^{\infty}|q(y, t)|^{4} d y\right)^{1 / 2}\left(\int_{0}^{\infty}\left|q_{x}(y, t)\right|^{2} d y\right)^{1 / 2} .
\end{aligned}
$$

So, under the assumptions of decaying initial data $q(0, t) \rightarrow 0$ as $t \rightarrow \infty$, and by Remark 2.3 , if $q(0, t), q_{t}(0, t)$ have polynomial decay of order $\mathcal{O}\left(t^{-\alpha}\right), \mathcal{O}\left(t^{-\beta}\right)$ respectively as $t \rightarrow \infty$, with $\alpha, \beta>1 / 2$, we have (cf. (2.13))

$$
\left\|q_{x}(\cdot, t)\right\| \leq c
$$

and thus, (3.24) yields

$$
\lim _{t \rightarrow \infty}\left|q^{3}(x, t)\right| \leq c \lim _{t \rightarrow \infty}\|q(\cdot, t)\|_{4}^{2}=0 .
$$

The relations involving the derivative in time follow from the NLS equation (1.1) (differentiating it in $x$ when needed) by using the fact that $q(x, \infty)=0$, and $\left(\partial_{x}^{k} q\right)(x, \infty)=0$.

Here, we note that by Remark 2.3, we have already proved that $q(x, t)$ is bounded uniformly in $x, t>0$. 
Now, we can prove sharper estimates than these of Remark 2.3 for $q$ and $q_{x}$ in the $L^{2}(0, \infty)$ norm in space. This is achieved by using the fact that the solution decays as $t \rightarrow \infty$ and the polynomial decay of the Dirichlet data for large times.

Theorem 3.4. Under the assumptions of Theorem 3.1 it holds that there exists $c>0$ independent of $t$ such that for any $t \geq 0$

$$
\|q(\cdot, t)\|^{2} \leq c\left(\int_{t}^{\infty}|q(0, r)|^{2} d r\right)^{1 / 2}
$$

and

$$
\left\|q_{x}(\cdot, t)\right\|^{2} \leq c\left(\int_{t}^{\infty}\left|q_{t}(0, r)\right|^{2} d r\right)^{1 / 2} .
$$

Proof. Integrating (2.4) in $(t, \infty)$ and using that by Proposition $3.3\|q(\cdot, \infty)\|=0$, we obtain

$$
\|q(\cdot, t)\|^{2}=-2 \int_{t}^{\infty} \operatorname{Im}\left\{q_{x}(0, r) \bar{q}(0, r)\right\} d r
$$

which yields for any $t \geq 0$

$$
\begin{aligned}
\|q(\cdot, t)\|^{2} & \leq c\left(\int_{0}^{\infty}\left|q_{x}(0, r)\right|^{2} d r\right)^{1 / 2}\left(\int_{t}^{\infty}|q(0, r)|^{2} d r\right)^{1 / 2} \\
& \leq c\left(\int_{t}^{\infty}|q(0, r)|^{2} d r\right)^{1 / 2},
\end{aligned}
$$

where we used (2.11). Here, $c>0$ is a constant independent of $t$. Thus, (3.26) holds true.

Furthermore, (2.6) when integrated in $(t, \infty)$ gives

$$
\left\|q_{x}(\cdot, t)\right\|^{2}=2 \int_{t}^{\infty} \operatorname{Re}\left\{q_{x}(0, r) \bar{q}_{t}(0, r)\right\} d r-\|q(\cdot, t)\|_{4}^{4},
$$

where we used that by Proposition $3.3\|q(\cdot, \infty)\|=0$ and $\left\|q_{x}(\cdot, \infty)\right\|=0$. Thus, for any $t \geq 0$ we have

$$
\left\|q_{x}(\cdot, t)\right\|^{2} \leq c\left(\int_{t}^{\infty}\left|q_{t}(0, r)\right|^{2} d r\right)^{1 / 2}
$$

since (2.11) is true. Here again, $c>0$ is a constant independent of $t$. So, (3.27) follows.

The next proposition presents an estimate for the Neumann data in $L^{2}(t, \infty)$ in terms of the Dirichlet data and its derivative on $(t, \infty)$. This result, similarly to the previous theorem connects the aforementioned quantities and is useful when large times decay for $q(0, t)$ and $q_{t}(0, t)$ is taken into account.

Proposition 3.5. Under the assumptions of Theorem 3.1 it holds that there exists $c>0$ independent of $t$ such that for any $t \geq 0$

$$
\begin{aligned}
\int_{t}^{\infty}\left|q_{x}(0, r)\right|^{2} d r \leq & c\left(\int_{t}^{\infty}|q(0, r)|^{2} d r\right)^{1 / 4}\left(\int_{t}^{\infty}\left|q_{t}(0, r)\right|^{2} d r\right)^{1 / 4} \\
& +c\left(\int_{t}^{\infty}|q(0, r)|^{2} d r\right)^{1 / 2}\left(\int_{t}^{\infty}\left|q_{t}(0, r)\right|^{2} d r\right)^{1 / 2}+\int_{t}^{\infty}|q(0, r)|^{4} d r .
\end{aligned}
$$


Proof. We integrate (2.8) in time in $(t, \infty)$ and use that $q(x, \infty)=0$ for any $x \in(0, \infty)$, to arrive at

$$
\begin{aligned}
\int_{t}^{\infty}\left|q_{x}(0, r)\right|^{2} d r= & -\mathrm{i}\left(q(\cdot, t), q_{x}(\cdot, t)\right)+\mathrm{i} \int_{t}^{\infty} q(0, r) \bar{q}_{t}(0, r) d r+\int_{t}^{\infty}|q(0, r)|^{4} d r \\
\leq & c\left(\int_{t}^{\infty}|q(0, r)|^{2} d r\right)^{1 / 4}\left(\int_{t}^{\infty}\left|q_{t}(0, r)\right|^{2} d r\right)^{1 / 4} \\
& +c\left(\int_{t}^{\infty}|q(0, r)|^{2} d r\right)^{1 / 2}\left(\int_{t}^{\infty}\left|q_{t}(0, r)\right|^{2} d r\right)^{1 / 2}+\int_{t}^{\infty}|q(0, r)|^{4} d r
\end{aligned}
$$

where we applied the estimates (3.26) and (3.27).

Now we have the following theorem.

Theorem 3.6. Under the assumptions of Theorem 3.1, for $\alpha>5 / 2, \beta>5 / 2$, and if as $t \rightarrow \infty$

$$
q_{t t}(0, t)=\mathcal{O}\left(t^{-\gamma}\right), \quad \gamma>1 / 2,
$$

then

$$
\int_{0}^{\infty}\left|q_{x t}(0, t)\right|^{2} d t<\infty
$$

Proof. Let

$$
v:=q_{t},
$$

then the NLS equation (1.1) gives by taking the derivative in $t$

$$
\mathrm{i} v_{t}+v_{x x}-4|q|^{2} v-2 q^{2} \bar{v}=0 .
$$

We multiply the above with $\overline{v_{x}}$ and integrate in space, to obtain after taking real parts

$$
\begin{aligned}
& \frac{\mathrm{i}}{2} \frac{d}{d t}\left(v(\cdot, t), v_{x}(\cdot, t)\right)+\frac{\mathrm{i}}{2} v(0, t) \bar{v}_{t}(0, t)-\frac{1}{2}\left|v_{x}(0, t)\right|^{2} \\
& -4 \operatorname{Re}\left(|q(\cdot, t)|^{2} v(\cdot, t), v_{x}(\cdot, t)\right)-2 \operatorname{Re}\left(q^{2}(\cdot, t) \bar{v}(\cdot, t), v_{x}(\cdot, t)\right)=0 .
\end{aligned}
$$

We integrate the above in time in $(0, t)$ and arrive at

$$
\begin{aligned}
\int_{0}^{t}\left|v_{x}(0, r)\right|^{2} d r= & \mathrm{i}\left(v(\cdot, r), v_{x}(\cdot, r)\right)+\mathrm{i} \int_{0}^{t} v(0, r) \bar{v}_{r}(0, r) d r \\
& -8 \int_{0}^{t} \operatorname{Re}\left(|q(\cdot, r)|^{2} v(\cdot, r), v_{x}(\cdot, r)\right) d r-4 \int_{0}^{t} \operatorname{Re}\left(q^{2}(\cdot, r) \bar{v}(\cdot, r), v_{x}(\cdot, r)\right) d r .
\end{aligned}
$$

But it holds that for any $x$

$$
\begin{aligned}
|q(x, r)|^{2} & \leq\|q(\cdot, r)\|\left\|q_{x}(\cdot, r)\right\| \\
& \leq c\left(\int_{r}^{\infty}|q(0, s)|^{2} d s\right)^{1 / 4}\left(\int_{r}^{\infty}\left|q_{s}(0, s)\right|^{2} d s\right)^{1 / 4}=: G(r),
\end{aligned}
$$

where we used (3.26) and (3.27). So, we get

$$
\max _{x \in(0, \infty)}|q(x, r)|^{2} \leq G(r) .
$$


Using (3.32) in (3.31), we have

$$
\begin{aligned}
\int_{0}^{t}\left|v_{x}(0, r)\right|^{2} d r= & \int_{0}^{t}\left|q_{t x}(0, r)\right|^{2} d r \\
\leq & c\|v(\cdot, t)\|\left\|v_{x}(\cdot, t)\right\|+c \int_{0}^{t}\left|v(0, r) \| v_{r}(0, r)\right| d r \\
& +c \int_{0}^{t} G(r)\|v(\cdot, r)\|\left\|v_{x}(\cdot, r)\right\| d r \\
\leq & c\|v(\cdot, t)\|^{2}+\hat{c_{0}}\left\|v_{x}(\cdot, t)\right\|^{2}+c \int_{0}^{t}\left|v(0, r) \| v_{r}(0, r)\right| d r \\
& +c \max _{r \geq 0}\left[\|v(\cdot, r)\|^{2}+\hat{c_{1}}\left\|v_{x}(\cdot, r)\right\|^{2}\right] \int_{0}^{t} G(r) d r,
\end{aligned}
$$

where $\hat{c_{0}}$ and $\hat{c_{1}}$ can be taken as small as we want (we will specify how small later).

We shall estimate $\|v(\cdot, r)\|,\left\|v_{x}(\cdot, r)\right\|$. We multiply (3.30) by $\bar{v}$ and integrate in space to obtain

$$
\begin{aligned}
\frac{d}{d t}\|v(\cdot, r)\|^{2} & =2 \operatorname{Im}\left\{v_{x}(0, r) \bar{v}(0, r)\right\}+4 \operatorname{Im}\left(q^{2}(\cdot, r) \bar{v}(\cdot, r), v(\cdot, r)\right) \\
& \leq c\left|v_{x}(0, r)\|v(0, r) \mid+c G(r)\| v(\cdot, r) \|^{2} .\right.
\end{aligned}
$$

But we observe that for $t \leq a_{1}, a_{1}$ large, then

$$
\int_{0}^{t} G(r) d r \leq \int_{0}^{a_{1}} G(r) d r \leq a_{1} \max _{r \in\left(0, a_{1}\right)} G(r)=a_{1} G(0) \leq c
$$

while for any $t>a_{1}$

$$
\begin{aligned}
\int_{0}^{t} G(r) d r=\int_{0}^{a_{1}} G(r) d r+\int_{a_{1}}^{t} G(r) d r & \leq c+c \int_{a_{1}}^{t}\left[\left(\int_{r}^{\infty}|q(0, s)|^{2} d s\right)^{1 / 4}\left(\int_{r}^{\infty}\left|q_{s}(0, s)\right|^{2} d s\right)^{1 / 4}\right] d r \\
& \leq c+c t^{\frac{-\alpha-\beta+3}{2}} \leq c,
\end{aligned}
$$

where we used that $\alpha+\beta>3$. Here, we used also that for $t>a_{1}$ then $q(0, t) \sim \mathcal{O}\left(t^{-\alpha}\right)$ and $q_{t}(0, t) \sim \mathcal{O}\left(t^{-\beta}\right)$. Thus, for any $t \geq 0$ we obtain

$$
e^{\int_{0}^{t} G(r) d r} \leq c
$$

uniformly in $t$, and as a result, Gronwall's lemma, applied to (3.34), gives

$$
\|v(\cdot, t)\|^{2} \leq c \int_{0}^{t}\left|v_{x}(0, r) \| v(0, r)\right| d r
$$

since $v(x, 0)=0$. So, we have for $c_{0}>0$ as small we want

$$
\max _{t \in[0, \infty)}\|v(\cdot, t)\|^{2} \leq c_{0} \int_{0}^{\infty}\left|v_{x}(0, r)\right|^{2} d r+c \int_{0}^{\infty}\left|q_{r}(0, r)\right|^{2} d r .
$$

In addition, (3.30) multiplied by $\bar{v}_{t}$ and integrated in space gives

$$
\begin{aligned}
& -\frac{1}{2} \frac{d}{d t}\left\|v_{x}(\cdot, r)\right\|^{2}-\operatorname{Re}\left\{v_{x}(0, r) \bar{v}_{r}(0, r)\right\} \\
& -4 \operatorname{Re}\left(|q(\cdot, r)|^{2} v(\cdot, r), v_{t}(\cdot, r)\right)-2 \operatorname{Re}\left(q^{2}(\cdot, r) \bar{v}(\cdot, r), v_{t}(\cdot, r)\right)=0 .
\end{aligned}
$$


We use now, cf. [15], that

$$
2 \operatorname{Re}\left(q^{2} \bar{v}, v_{t}\right)=\frac{d}{d t} \operatorname{Re}\left(q^{2}, v^{2}\right)+\mathcal{A}, \quad 4 \operatorname{Re}\left(|q|^{2} v, v_{t}\right)=2 \frac{d}{d t} \operatorname{Re}\left(|q|^{2},|v|^{2}\right)+\mathcal{B},
$$

for

$$
|\mathcal{A}|+|\mathcal{B}| \leq c \int_{0}^{\infty}|q|\left|q_{t}\right||v|^{2} d x=c \int_{0}^{\infty}|q \| v|^{3} d x
$$

So, we get

$$
\begin{aligned}
|\mathcal{A}+\mathcal{B}| & \leq c \int_{0}^{\infty}|q\|v\| v|^{2} d x \leq c \int_{0}^{\infty}\left|q\left\|v \mid\left(\|v\|\left\|v_{x}\right\|\right) d x \leq c\right\| q\|\| v\left\|^{2}\right\| v_{x} \|\right. \\
& \leq c\|q\|\|v\|^{4}+c\|q\|\left\|v_{x}\right\|^{2} .
\end{aligned}
$$

Hence, (3.37) by using (3.38), yields

$$
\begin{aligned}
& \frac{d}{d t}\left\|v_{x}(\cdot, r)\right\|^{2}+2 \frac{d}{d t} \operatorname{Re}\left(q^{2}(\cdot, r), v^{2}(\cdot, r)\right)+4 \frac{d}{d t} \operatorname{Re}\left(|q(\cdot, r)|^{2},|v(\cdot, r)|^{2}\right) \\
\leq & c\left|v_{x}(0, r)\left\|v_{r}(0, r) \mid+c\right\| q(\cdot, r)\|\| v(\cdot, r)\left\|^{4}+c\right\| q(\cdot, r)\|\| v_{x}(\cdot, r) \|^{2}\right.
\end{aligned}
$$

Relation (3.26) yields

$$
\int_{0}^{t}\|q(\cdot, r)\| d r \leq \int_{0}^{a_{1}}\|q(\cdot, r)\| d r \leq c
$$

for $t \leq a_{1}$, while for $t>a_{1}$

$$
\begin{aligned}
\int_{0}^{t}\|q(\cdot, r)\| d r & \leq c \int_{0}^{t}\left(\int_{s}^{\infty}|q(0, r)|^{2} d r\right)^{1 / 4} d s \leq c+c \int_{a_{1}}^{t}\left(\int_{s}^{\infty}|q(0, r)|^{2} d r\right)^{1 / 4} d s \\
& \leq c+c t^{\frac{-2 \alpha+1}{4}+1} \leq c,
\end{aligned}
$$

since $\alpha>5 / 2$. Thus, for any $t \geq 0$, Gromwall's lemma in (3.39) gives (since $v_{x}(x, 0)=q_{t x}(x, 0)=0$ and $v(x, 0)=0$ )

$$
\begin{aligned}
\left\|v_{x}(\cdot, t)\right\|^{2} \leq & c\left\|q^{2}(\cdot, t)\right\|\left\|v^{2}(\cdot, t)\right\|+c \int_{0}^{t}\left[\int_{0}^{\infty}|q(x, r)|^{2}|v(x, r)|^{2} d x\right] d r \\
& +c \int_{0}^{t}\left|v_{x}(0, r)\left\|v_{r}(0, r) \mid d r+\int_{0}^{t} G_{1}(r)\right\| v(\cdot, r) \|^{4} d r\right. \\
\leq & c\left\|q^{2}(\cdot, t)\right\|\left\|v^{2}(\cdot, t)\right\|+c \int_{0}^{t} \max _{x \geq 0}\left(|q(x, r)|^{2}\right)\|v(\cdot, r)\|^{2} d r \\
& +c \int_{0}^{t}\left|v_{x}(0, r)\left\|v_{r}(0, r) \mid d r+c \int_{0}^{t} G_{1}(r)\right\| v(\cdot, r) \|^{4} d r\right. \\
\leq & c\left\|q^{2}(\cdot, t)\right\|\left\|v^{2}(\cdot, t)\right\|+c \int_{0}^{t} \max _{x \geq 0}\left(|q(x, r)|^{2}\right) 1 d r+c \int_{0}^{t} \max \left(|q(x, r)|^{2}\right)\|v(\cdot, r)\|^{4} d r \\
& +c \int_{0}^{t}\left|v_{x}(0, r)\left\|v_{r}(0, r) \mid d r+c \int_{0}^{t} G_{1}(r)\right\| v(\cdot, r) \|^{4} d r\right. \\
\leq & c\left\|q^{2}(\cdot, t)\right\|\left\|v^{2}(\cdot, t)\right\|+c \\
& +c \int_{0}^{t}\left|v_{x}(0, r)\left\|v_{r}(0, r) \mid d r+c \int_{0}^{t}\left[G(r)+G_{1}(r)\right]\right\| v(\cdot, r) \|^{4} d r\right.
\end{aligned}
$$


since

$$
\|q(\cdot, s)\| \leq c\left(\int_{s}^{\infty}|q(0, r)|^{2} d r\right)^{1 / 4}=: G_{1}(s)
$$

while as before, for the same definition of $G$ (see (3.32) $)$,

$$
\begin{aligned}
|q(x, r)|^{2} & \leq\|q(\cdot, r)\|\left\|q_{x}(\cdot, r)\right\| \\
& \leq c\left(\int_{r}^{\infty}|q(0, s)|^{2} d s\right)^{1 / 4}\left(\int_{r}^{\infty}\left|q_{s}(0, s)\right|^{2} d s\right)^{1 / 4}=: G(r),
\end{aligned}
$$

and thus,

Furthermore, we have

$$
\int_{0}^{t} \max _{x \geq 0}\left(|q(x, r)|^{2}\right) d r \leq \int_{0}^{t} G(r) d r \leq c
$$

$$
\left\|v^{2}\right\| \leq c\|v\|^{3 / 2}\left\|v_{x}\right\|^{1 / 2} \leq c_{1}\left\|v_{x}\right\|+c\|v\|^{3} \leq c_{1}\left\|v_{x}\right\|^{2}+c\|v\|^{4}+c,
$$

where $c_{1}>0$ can be chosen as small as we want. Also, the same argument together with (3.26), (3.27), gives

$$
\left\|q^{2}(\cdot, r)\right\| \leq c\left\|q_{x}(\cdot, r)\right\|^{2}+c\|q(\cdot, r)\|^{4}+c \leq c,
$$

uniformly for any $r$. So, using (3.41) and (3.42) in (3.40), we obtain

$$
\begin{aligned}
\left\|v_{x}(\cdot, t)\right\|^{2} \leq & c\left(c_{1}\left\|v_{x}(\cdot, t)\right\|^{2}+c\|v(\cdot, t)\|^{4}+c\right)+c+c \int_{0}^{t}\left[G(r)+G_{1}(r)\right]\|v(\cdot, r)\|^{4} d r \\
& +c \int_{0}^{t}\left|v_{x}(0, r)\right|^{2} d r+c \int_{0}^{t}\left|v_{r}(0, r)\right|^{2} d r \\
\leq & c c_{1}\left\|v_{x}(\cdot, t)\right\|^{2}+c\|v(\cdot, t)\|^{4}+c \int_{0}^{t}\left[G(r)+G_{1}(r)\right]\|v(\cdot, r)\|^{4} d r \\
& +c \int_{0}^{t}\left|v_{x}(0, r)\right|^{2} d r+c \int_{0}^{t}\left|v_{r}(0, r)\right|^{2} d r+c .
\end{aligned}
$$

Furthermore, by (3.35) we arrive at

$$
\begin{aligned}
\|v(\cdot, t)\|^{4} & \leq c\left(\int_{0}^{t}\left|v_{x}(0, r)\right||v(0, r)| d r\right)^{2} \leq c\left(\left(\int_{0}^{t}\left|v_{x}(0, r)\right|^{2} d r\right)^{1 / 2}\left(\int_{0}^{t}|v(0, r)|^{2} d r\right)^{1 / 2}\right)^{2} \\
& \leq c\left(\int_{0}^{t}\left|v_{x}(0, r)\right|^{2} d r\right)\left(\int_{0}^{t}|v(0, r)|^{2} d r\right) .
\end{aligned}
$$

Thus, we have

$$
\|v(\cdot, t)\|^{4} \leq c \int_{0}^{t}\left|v_{x}(0, r)\right|^{2} d r
$$

while

$$
\begin{aligned}
\int_{0}^{t}\left[G(r)+G_{1}(r)\right]\|v(\cdot, r)\|^{4} d r & \leq \max _{t \geq 0}\|v(\cdot, r)\|^{4} \int_{0}^{t}\left[G(r)+G_{1}(r)\right] d r \\
& \leq c \int_{0}^{\infty}\left|v_{x}(0, r)\right|^{2} d r \int_{0}^{t}\left[G(r)+G_{1}(r)\right] d r \leq c \int_{0}^{\infty}\left|v_{x}(0, r)\right|^{2} d r
\end{aligned}
$$


since $\alpha+\beta>3$ and $\alpha>5 / 2$ (note that

$$
\int_{0}^{t} G_{1}(r) d r \leq c+c \int_{a_{1}}^{t} r^{\frac{-2 \alpha+1}{4}} d r \leq c
$$

for $\alpha>5 / 2$ ). Therefore, using (3.44) and (3.45) in (3.43), we obtain

$$
\left\|v_{x}(\cdot, t)\right\|^{2} \leq c c_{1}\left\|v_{x}(\cdot, t)\right\|^{2}+c \int_{0}^{\infty}\left|v_{x}(0, r)\right|^{2} d r+c \int_{0}^{t}\left|v_{r}(0, r)\right|^{2} d r+c
$$

and so, if $c_{1}$ is chosen small enough,

$$
\left\|v_{x}(\cdot, t)\right\|^{2} \leq c \int_{0}^{\infty}\left|v_{x}(0, r)\right|^{2} d r+c \int_{0}^{t}\left|v_{r}(0, r)\right|^{2} d r+c
$$

Observe now that since $\int_{0}^{\infty} G(r) d r<\infty$, then (3.33) gives

$$
\begin{aligned}
\int_{0}^{t}\left|v_{x}(0, r)\right|^{2} d r \leq & c\|v(\cdot, r)\|^{2}+\hat{c_{0}}\left\|v_{x}(\cdot, r)\right\|^{2}+c \int_{0}^{t}\left|v(0, r) \| v_{r}(0, r)\right| d r \\
& +\max _{r \geq 0}\left[\|v(\cdot, r)\|^{2}+\hat{c_{1}}\left\|v_{x}(\cdot, r)\right\|^{2}\right] .
\end{aligned}
$$

and thus, relations (3.36) and (3.46) yield

$$
\begin{aligned}
\int_{0}^{t}\left|v_{x}(0, r)\right|^{2} d r \leq & c\left(c_{0} \int_{0}^{\infty}\left|v_{x}(0, r)\right|^{2} d r+c \int_{0}^{\infty}\left|q_{r}(0, r)\right|^{2} d r\right) \\
& +c\left(\hat{c_{0}} \int_{0}^{\infty}\left|v_{x}(0, r)\right|^{2} d r+\hat{c_{1}} \int_{0}^{\infty}\left|v_{x}(0, r)\right|^{2} d r+c \int_{0}^{\infty}\left|v_{r}(0, r)\right|^{2} d r+c\right) \\
& +c \int_{0}^{\infty}|v(0, r)|\left|v_{r}(0, r)\right| d r .
\end{aligned}
$$

Thus, if $c_{0}, c_{2}$ and $\hat{c_{0}}, \hat{c_{1}}$ are chosen appropriately small, we arrive at

$$
\int_{0}^{\infty}\left|v_{x}(0, r)\right|^{2} d r \leq c \int_{0}^{\infty}\left|q_{r}(0, r)\right|^{2} d r+c \int_{0}^{\infty}\left|q_{r r}(0, r)\right|^{2} d r+c \leq c,
$$

since $\beta>1 / 2$ and $\gamma>1 / 2$.

Remark 3.7. For the previous proof, the crucial argument was the application of Gronwall's lemma for $t \in \mathbb{R}^{+}$. This could in general give exponentially growing coefficients in time; in our case the proven decay of solution for large times leads to the validity of the estimates (3.26) and (3.27) which results to uniformly bounded coefficients.

Next we prove the following general theorem that connects the polynomial decay of Dirichlet data to an analogous resulting decay of the Neumann one.

Theorem 3.8. Let $q$ be the unique global classical solution $q \in C^{1}\left(L^{2}\right) \cap C^{0}\left(H^{2}\right)$ of the problem (1.1) -(1.2), with $Q \in C^{2}$ and $Q(0)=0$, with $\lambda=1$, under the assumption (2.2). If as $t \rightarrow \infty$

$$
q(0, t)=\mathcal{O}\left(t^{-\alpha}\right), \quad q_{t}(0, t)=\mathcal{O}\left(t^{-\beta}\right), \quad q_{t t}(0, t)=\mathcal{O}\left(t^{-\gamma}\right)
$$

where

$$
\alpha>5 / 2, \quad \beta>5 / 2, \quad \gamma>1 / 2,
$$

then there exists $c>0$ independent of $t$ such that for any $t$ large

$$
\left|q_{x}(0, t)\right| \leq c t^{-\delta}
$$


for $\delta=\min \left\{\frac{\alpha+\beta-1}{2}, \frac{4 \alpha-1}{4}\right\}$.

Proof. Consider $t \geq 0$ large and $s$ large such that $s<t$, then by using first Theorem 3.6 and then Proposition 3.5, we have

$$
\begin{aligned}
\left|q_{x}(0, t)\right| \leq & c\left(\int_{s}^{\infty}\left|q_{x}(0, r)\right|^{2} d r\right)^{1 / 4}\left(\int_{s}^{\infty}\left|q_{x r}(0, r)\right|^{2} d r\right)^{1 / 4} \\
\leq & c\left(\int_{s}^{\infty}\left|q_{x}(0, r)\right|^{2} d r\right)^{1 / 4}\left(\int_{0}^{\infty}\left|q_{x r}(0, r)\right|^{2} d r\right)^{1 / 4} \\
\leq & c\left(\int_{s}^{\infty}\left|q_{x}(0, r)\right|^{2} d r\right)^{1 / 4} \\
\leq & c\left[\left(\int_{s}^{\infty}|q(0, r)|^{2} d r\right)^{1 / 4}\left(\int_{s}^{\infty}\left|q_{t}(0, r)\right|^{2} d r\right)^{1 / 4}\right. \\
& \left.+\left(\int_{s}^{\infty}|q(0, r)|^{2} d r\right)^{1 / 2}\left(\int_{s}^{\infty}\left|q_{t}(0, r)\right|^{2} d r\right)^{1 / 2}+\int_{s}^{\infty}|q(0, r)|^{4} d r\right]^{1 / 4} \\
\leq & c\left[s^{\frac{-2 \alpha+1}{4}} s^{\frac{-2 \beta+1}{4}}+s^{\frac{-2 \alpha+1}{2}} s^{\frac{-2 \beta+1}{2}}+s^{\frac{-4 \alpha+1}{4}}\right] \\
\leq & {\left[s^{\frac{-\alpha-\beta+1}{2}}+s^{-\alpha-\beta+1}+s^{\frac{-4 \alpha+1}{4}}\right] } \\
\leq & {\left[s^{\frac{-\alpha-\beta+1}{2}}+s^{\frac{-4 \alpha+1}{4}}\right] \leq c s^{-\delta}, }
\end{aligned}
$$

for

$$
\delta=\min \left\{\frac{\alpha+\beta-1}{2}, \frac{4 \alpha-1}{4}\right\} .
$$

So, we obtain

$$
\lim _{t \rightarrow s^{-}}\left|q_{x}(0, t)\right|=\left|q_{x}(0, s)\right| \leq c s^{-\delta}
$$

which gives the result.

In particular, if the Dirichlet data $Q$ belong in the Schwartz class, then the Neumann values $q_{x}(0, t)$ also belong in the Schwartz class.

An immediate corollary is the following.

Theorem 3.9. Let $q$ be the unique global classical solution $q \in C^{1}\left(L^{2}\right) \cap C^{0}\left(H^{2}\right)$ of the problem (1.1)-(1.2), with $Q \in C^{2}$ and $Q(0)=0$, with $\lambda=1$, under the assumption (2.2). If as $t \rightarrow \infty$

$$
q(0, t)=\mathcal{O}\left(t^{-5 / 2-\varepsilon}\right), \quad q_{t}(0, t)=\mathcal{O}\left(t^{-5 / 2-\varepsilon}\right), \quad q_{t t}(0, t)=\mathcal{O}\left(t^{-1 / 2-\varepsilon}\right),
$$

for some small $\varepsilon>0$, then there exists $c>0$ independent of $t$ such that for any $t$ large

$$
\left|q_{x}(0, t)\right| \leq c t^{-2-\varepsilon} \text {. }
$$

In particular

$$
\int_{0}^{\infty}\left|q_{x}(0, t)\right| d t<\infty
$$

As a result, the assumptions of the unified method of scattering and inverse scattering of Fokas et al. are now rigorously justified for the model case of defocusing NLS. 
Remark 3.10. Unlike KdV and the associated Schrödinger operator, scattering and inverse scattering for the NLS and the Dirac operator has not been so thoroughly investigated in the literature. In particular, the question of the optimal data for the inverse scattering method to work has never been fully considered.

Our Theorems 3.9 (and 4.5 in the next section) are motivated by the analysis in [17] which implies that an $L^{1}$ assumption for $Q, Q_{t}$ and $q_{x}(0, t)$ is enough for the inverse scattering method to solve the initial value problem. Indeed, in [17] an explicit formula for the eigenfunction for the second Lax operator (the t-problem) is provided. ( 17] does not consider infinite times, but a similar formula is possible for our purposes.) This formula involves the solution of a Goursat type problem for a system of PDEs. An inspection of the formula and the system reveals [27] that indeed if $Q(t), Q_{t}(t)$ and $q_{x}(0, t)$ are $L^{1}(0, \infty)$ the eigenfunction for the second Lax operator and hence the scattering construction and the resulting Riemann-Hilbert problem are possible.

Of course, our Theorems 3.8 (and 4.4) are flexible enough to provide a sufficient (and reasonably weak) condition on the Dirichlet data that guarantees any required decay condition on the Neumann data.

An alternative approach appears in the Appendix. It is more general, but it only bounds the $L^{1}$-norm for $q_{x}(0, t)$ (and for $t q_{x}(0, t)$ ).

\section{Focusing NLS}

In this section we have to make the assumption that $q(x, t) \rightarrow 0$ as $t \rightarrow \infty$ pointwise. While this follows easily in the case of defocusing NLS from the 4-norm estimate, it is not clear how to prove it in the case of focusing NLS.

The following proposition holds true.

Proposition 4.1. Let $q$ be the unique global classical solution $q \in C^{1}\left(L^{2}\right) \cap C^{0}\left(H^{2}\right)$ of the problem (1.1)-(1.2), with $Q \in C^{2}$ and $Q(0)=0$, with $\lambda=-1$, under the assumption (2.2). Also let

$$
\int_{0}^{\infty}|q(0, t)|^{2} d t
$$

be sufficiently small. Then it holds that

$$
\|q(\cdot, t)\|^{2} \leq c\left(\int_{t}^{\infty}|q(0, r)|^{2} d r\right)^{1 / 2},
$$

and

$$
\left\|q_{x}(\cdot, t)\right\|^{2} \leq c\left(\int_{t}^{\infty}\left|q_{t}(0, r)\right|^{2} d r\right)^{1 / 2}+c\left(\int_{t}^{\infty}|q(0, r)|^{2} d r\right)^{3 / 2} .
$$

Proof. The first inequality is obvious since its proof is independent of the sign of the nonlinearity in the NLS equation, cf. the proof of (3.26).

Relation, (2.6) when integrated in $(t, \infty)$ gives

$$
\left\|q_{x}(\cdot, t)\right\|^{2}=2 \operatorname{Re} \int_{t}^{\infty} q_{x}(0, r) \bar{q}_{t}(0, r) d r+\|q(\cdot, t)\|_{4}^{4},
$$

Thus, using

$$
\|q\|_{4}^{4} \leq\|q\|^{3}\left\|q_{x}\right\| \leq \tilde{c}\left\|q_{x}\right\|^{2}+c\|q\|^{6},
$$

for $\tilde{c}>0$ as small as we need and (4.1), we obtain (4.2). The smallness of $\int_{0}^{\infty}|q(0, t)|^{2} d t$, is needed so that Theorem 2.1 holds true and thus $\int_{0}^{\infty}\left|q_{x}(0, r)\right|^{2} d r$ is bounded. 
Furthermore, we proceed by proving the next estimate.

Proposition 4.2. Under the assumptions of Proposition 4.1 it holds that there exists $c>0$ independent of $t$ such that for any $t \geq 0$

$$
\begin{aligned}
\int_{t}^{\infty}\left|q_{x}(0, r)\right|^{2} d r \leq & c\left(\int_{t}^{\infty}|q(0, r)|^{2} d r\right)^{1 / 4}\left(\int_{t}^{\infty}\left|q_{t}(0, r)\right|^{2} d r\right)^{1 / 4} \\
& +c\left(\int_{t}^{\infty}|q(0, r)|^{2} d r\right) \\
& +c\left(\int_{t}^{\infty}|q(0, r)|^{2} d r\right)^{1 / 2}\left(\int_{t}^{\infty}\left|q_{t}(0, r)\right|^{2} d r\right)^{1 / 2} .
\end{aligned}
$$

Proof. We integrate (2.8) in time in $(t, \infty)$ and use that $q(x, \infty)=0$ for any $x \in(0, \infty)$ to obtain the result.

Now, we can establish the following theorem.

Theorem 4.3. Under the assumptions of Proposition 4.1, if as $t \rightarrow \infty$

$$
q(0, t)=\mathcal{O}\left(t^{-\alpha}\right), \quad q_{t}(0, t)=\mathcal{O}\left(t^{-\beta}\right), \quad q_{t t}(0, t)=\mathcal{O}\left(t^{-\gamma}\right)
$$

where

$$
\alpha>5 / 2, \quad \beta>1 / 2, \quad \gamma>1 / 2,
$$

then

$$
\int_{0}^{\infty}\left|q_{x t}(0, t)\right|^{2} d t<\infty
$$

Proof. Let

$$
v:=q_{t},
$$

then the NLS equation (1.1) gives by taking the derivative in $t$

$$
\mathrm{i} v_{t}+v_{x x}+4|q|^{2} v+2 q^{2} \bar{v}=0 .
$$

We multiply the above with $\bar{v}_{x}$ and integrate in space, to obtain

$$
\begin{aligned}
& \frac{\mathrm{i}}{2} \frac{d}{d t}\left(v(\cdot, t), v_{x}(\cdot, t)\right)+\frac{\mathrm{i}}{2} v(0, t) \bar{v}_{t}(0, t)-\frac{1}{2}\left|v_{x}(0, t)\right|^{2} \\
& +4 \operatorname{Re}\left(|q(\cdot, t)|^{2} v(\cdot, t), v_{x}(\cdot, t)\right)+2 \operatorname{Re}\left(q^{2}(\cdot, t) \bar{v}(\cdot, t), v_{x}(\cdot, t)\right)=0 .
\end{aligned}
$$

We integrate the above in time in $(0, t)$ and get

$$
\begin{aligned}
\int_{0}^{t}\left|v_{x}(0, r)\right|^{2} d r= & \mathrm{i}\left(v(\cdot, r), v_{x}(\cdot, r)\right)+\mathrm{i} \int_{0}^{t} v(0, r) \bar{v}_{r}(0, r) d r \\
& +8 \int_{0}^{t} \operatorname{Re}\left(|q(\cdot, r)|^{2} v(\cdot, r), v_{x}(\cdot, r)\right) d r+4 \int_{0}^{t} \operatorname{Re}\left(q^{2}(\cdot, r) \bar{v}(\cdot, r), v_{x}(\cdot, r)\right) d r .
\end{aligned}
$$

But it holds that for any $x$

$$
\begin{aligned}
|q(x, r)|^{2} \leq & \|q(\cdot, r)\|\left\|q_{x}(\cdot, r)\right\| \\
\leq & c\left(\int_{r}^{\infty}|q(0, s)|^{2} d s\right)^{1 / 4}\left(\int_{r}^{\infty}\left|q_{s}(0, s)\right|^{2} d s\right)^{1 / 4} \\
& +c\left(\int_{r}^{\infty}|q(0, s)|^{2} d s\right)=: \tilde{G}(r) .
\end{aligned}
$$


The rest of the proof is identical to that of Theorem 4.3 with $\tilde{G}$ in place of $G$. Note that since $\alpha>5 / 2, \beta>1 / 2$, then we have

$$
\int_{0}^{\infty} \tilde{G}(r) d r<\infty
$$

Now, we have a theorem for the polynomial decay of the Neumann data in the case of focusing NLS.

Theorem 4.4. Let $q$ be the unique global classical solution $q \in C^{1}\left(L^{2}\right) \cap C^{0}\left(H^{2}\right)$ of the problem (1.1)-(1.2), with $Q \in C^{2}$ and $Q(0)=0$, with $\lambda=-1$, under the assumption (2.2). Also, let

$$
\int_{0}^{\infty}|q(0, t)|^{2} d t
$$

be sufficiently small. If as $t \rightarrow \infty$

$$
q(0, t)=\mathcal{O}\left(t^{-\alpha}\right), \quad q_{t}(0, t)=\mathcal{O}\left(t^{-\beta}\right), \quad q_{t t}(0, t)=\mathcal{O}\left(t^{-\gamma}\right),
$$

for

$$
\alpha>5 / 2, \quad \beta>1 / 2, \quad \gamma>1 / 2,
$$

then there exists $c>0$ independent of $t$ such that for any $t$ large

$$
\left|q_{x}(0, t)\right| \leq c t^{-\delta}
$$

for $\delta=\min \left\{\frac{\alpha+\beta-1}{2}, 2 \alpha-1\right\}$.

Proof. The proof is analogous to this of Theorem 3.8 .

Again, the following corollary is obvious.

Theorem 4.5. Let $q$ be the unique global classical solution $q \in C^{1}\left(L^{2}\right) \cap C^{0}\left(H^{2}\right)$ of the problem (1.1)-(1.2), with $Q \in C^{2}$ and $Q(0)=0$, with $\lambda=-1$, under the assumption (2.2). Also, let

$$
\int_{0}^{\infty}|q(0, t)|^{2} d t
$$

be sufficiently small. If as $t \rightarrow \infty$

$$
q(0, t)=\mathcal{O}\left(t^{-5 / 2-\varepsilon}\right), \quad q_{t}(0, t)=\mathcal{O}\left(t^{-5 / 2-\varepsilon}\right), \quad q_{t t}(0, t)=\mathcal{O}\left(t^{-1 / 2-\varepsilon}\right),
$$

for some small $\varepsilon>0$, then there exists $c>0$ independent of $t$ such that for any $t$ large

$$
\left|q_{x}(0, t)\right| \leq c t^{-2-\varepsilon}
$$

and thus,

$$
\int_{0}^{\infty}\left|q_{x}(0, t)\right| d t<\infty
$$

As a result, the decay assumption of the unified method of scattering and inverse scattering of Fokas et al. is now justified for the case of focusing NLS, at least under the stated assumptions.

We also note that if the Dirichlet data $Q$ belong in the Schwartz class, then the Neumann values $q_{x}(0, t)$ also belong in the Schwartz class. 


\section{Conclusions}

The main result of this paper is the following: in order to obtain fast enough decay for the Neumann data $q_{x}(0, t)$ so that the Fokas method is applicable, it is sufficient to impose a certain (mild) polynomial decay on the Dirichlet data $q(0, t)$ and its derivatives $q_{t}(0, t)$ and $q_{t t}(0, t)$.

Of course the decay assumed here for the Dirichlet data, although sufficient, may not be the optimal one.

For the focusing NLS the answer is only provided under the assumption that the solution tends to 0 as $t \rightarrow \infty$ and a smallness assumption for $\int_{0}^{\infty}|q(0, t)|^{2} d t$. The general problem remains open for this case, as for the moment we do not posses a proof of this decay.

\section{Appendix A. On the $L^{1}$ estimate}

We present for completeness an alternative and more general approach for deriving decay estimates and the $L^{1}$ estimate for the Neumann data.

Using that for all positive $x$,

$$
\lim _{t \rightarrow \infty} q(x, t)=0,
$$

(proved for the defocusing NLS, cf. (3.25), assuming as we did in section 4 that it is true for the focusing NLS as well) we can prove a general $L^{1}(0, \infty)$ bound for $q_{x}(0, t)$, for both cases, as follows.

Consider the relation (2.8) (true for both the defocusing and focusing NLS)

$$
\left|q_{x}(0, t)\right|^{2}=\mathrm{i} \frac{d}{d t}\left(q, q_{x}\right)+\mathrm{i} q(0, t) \bar{q}_{t}(0, t)+\lambda|q(0, t)|^{4} .
$$

Take $p>1$ and multiply the above with $t^{p}$ to obtain

$$
t^{p}\left|q_{x}(0, t)\right|^{2}=\mathrm{i} t^{p} \frac{d}{d t}\left(q, q_{x}\right)+\mathrm{i} t^{p} q(0, t) \bar{q}_{t}(0, t)+\lambda t^{p}|q(0, t)|^{4} .
$$

Integration of (A.2) in time gives

$$
\begin{aligned}
\int_{0}^{\infty} t^{p}\left|q_{x}(0, t)\right|^{2} d t= & \mathrm{i} \int_{0}^{\infty} t^{p} \frac{d}{d t}\left(q(\cdot, t), q_{x}(\cdot, t)\right) d t+\mathrm{i} \int_{0}^{\infty} t^{p} q(0, t) \bar{q}_{t}(0, t) d t+\lambda \int_{0}^{\infty} t^{p}|q(0, t)|^{4} d t \\
= & \mathrm{i}\left[t^{p}\left(q(\cdot, t), q_{x}(\cdot, t)\right)\right]_{0}^{\infty}-\mathrm{i} \int_{0}^{\infty} p t^{p-1}\left(q(\cdot, t), q_{x}(\cdot, t)\right) d t \\
& +\mathrm{i} \int_{0}^{\infty} t^{p} q(0, t) \bar{q}_{t}(0, t) d t+\lambda \int_{0}^{\infty} t^{p}|q(0, t)|^{4} d t
\end{aligned}
$$

and thus

$$
\begin{aligned}
\int_{0}^{\infty} t^{p}\left|q_{x}(0, t)\right|^{2} d t \leq & \lim _{t \rightarrow \infty}\left(t^{p}\|q(\cdot, t)\|\left\|q_{x}(\cdot, t)\right\|\right)+c \int_{0}^{\infty} t^{p-1}\|q(\cdot, t)\|\left\|q_{x}(\cdot, t)\right\| d t \\
& +c \int_{0}^{\infty} t^{p}\left|q(0, t) \| q_{t}(0, t)\right| d t+\lambda \int_{0}^{\infty} t^{p}|q(0, t)|^{4} d t .
\end{aligned}
$$

Since under the assumptions of Theorem 3.1 it holds (cf. the proof of (3.26) ) that there exists $c>0$ independent of $t$ such that for any $t \geq 0$

$$
\|q(\cdot, t)\|^{2} \leq c\left(\int_{t}^{\infty}|q(0, r)|^{2} d r\right)^{1 / 2}
$$

while $\left\|q_{x}\right\|$ is bounded uniformly in $t$, we obtain by (A.3) 


$$
\begin{aligned}
\int_{0}^{\infty} t^{p}\left|q_{x}(0, t)\right|^{2} d t \leq & c \lim _{t \rightarrow \infty}\left(t^{p}\left(\int_{t}^{\infty}|q(0, r)|^{2} d r\right)^{1 / 4}\right) \\
& +c \int_{0}^{\infty} t^{p-1}\left(\int_{t}^{\infty}|q(0, r)|^{2} d r\right)^{1 / 4} d t \\
& +c \int_{0}^{\infty} t^{p}|q(0, t)|\left|q_{t}(0, t)\right| d t+\lambda \int_{0}^{\infty} t^{p}|q(0, t)|^{4} d t
\end{aligned}
$$

Furthermore,

$$
t^{p}\|q(\cdot, t)\|\left\|q_{x}(\cdot, t)\right\| \leq c t^{p}\left(\int_{t}^{\infty}|q(0, r)|^{2} d r\right)^{1 / 4} \rightarrow 0
$$

if $q(0, t)$ has a sufficiently fast (polynomial) decay as $t \rightarrow \infty$. Also, we have

$$
\int_{0}^{\infty} t^{p-1}\|q(\cdot, t)\|\left\|q_{x}(\cdot, t)\right\| d t \leq c \int_{0}^{\infty} t^{p-1}\left(\int_{t}^{\infty}|q(0, r)|^{2} d r\right)^{1 / 4} d t \leq c
$$

if again $q(0, t)$ has a sufficiently fast decay as $t \rightarrow \infty$. The same argument of sufficiently fast decay for $q_{t}(0, t)$ as $t \rightarrow \infty$, together with the previous one, finally gives, using (A.4)

$$
\int_{0}^{\infty} t^{p}\left|q_{x}(0, t)\right|^{2} d t \leq c
$$

as $p>1$.

Note that a weaker assumption on initial data $q(0, t)$ and $q_{t}(0, t)$ resulting in a bounded righthand side of relation (A.4) also gives (A.5). This observation makes this approach more general.

Now, we are ready to derive the $L^{1}(0, \infty)$ estimate for $\left|q_{x}(0, t)\right|$. Indeed,

$$
\begin{aligned}
\int_{0}^{\infty}\left|q_{x}(0, t)\right| d t & =\int_{0}^{1}\left|q_{x}(0, t)\right| d t+\int_{1}^{\infty} t^{-\frac{1}{2}-\frac{\varepsilon}{2}} t^{\frac{1}{2}+\frac{\varepsilon}{2}}\left|q_{x}(0, t)\right| d t \\
& \leq c\left(\int_{0}^{1} 1^{2} d t\right)^{1 / 2}\left(\int_{0}^{1}\left|q_{x}(0, t)\right|^{2} d t\right)^{1 / 2}+\left(\int_{1}^{\infty} t^{-1-\varepsilon} d t\right)^{1 / 2}\left(\int_{1}^{\infty} t^{1+\varepsilon}\left|q_{x}(0, t)\right|^{2} d t\right)^{1 / 2} \\
& \leq c+c\left(\int_{0}^{\infty} t^{1+\varepsilon}\left|q_{x}(0, t)\right|^{2} d t\right)^{1 / 2} \leq c
\end{aligned}
$$

where we used (A.5) for $p:=1+\varepsilon$.

Remark A.1. We could, in fact, proceed to a detailed presentation of the sufficient order of decay of $q(0, t)$ and $q_{t}(0, t)$ which will guarantee a required order of decay of $q_{x}(0, t)$, as we did in Sections 3 and 4 . We leave the tedious calculations to the interested reader. 
Remark A.2. It is easy to also derive the $L^{1}(0, \infty)$ condition for $t\left|q_{x}(0, t)\right|$, if one wishes. As in (A.6), we have

(A.7)

$$
\begin{aligned}
\int_{0}^{\infty} t\left|q_{x}(0, t)\right| d t & =\int_{0}^{1} t\left|q_{x}(0, t)\right| d t+\int_{1}^{\infty} t t^{-\frac{1}{2}-\frac{\varepsilon}{2}} t^{\frac{1}{2}+\frac{\varepsilon}{2}}\left|q_{x}(0, t)\right| d t \\
& \leq c\left(\int_{0}^{1} t^{2} d t\right)^{1 / 2}\left(\int_{0}^{1}\left|q_{x}(0, t)\right|^{2} d t\right)^{1 / 2}+\left(\int_{1}^{\infty} t^{-1-\varepsilon} d t\right)^{1 / 2}\left(\int_{1}^{\infty} t^{2} t^{1+\varepsilon}\left|q_{x}(0, t)\right|^{2} d t\right)^{1 / 2} \\
& \leq c+c\left(\int_{0}^{\infty} t^{3+\varepsilon}\left|q_{x}(0, t)\right|^{2} d t\right)^{1 / 2} \leq c
\end{aligned}
$$

where we used (A.5) for $p:=3+\varepsilon$.

Remark A.3. The approach of the Appendix is more general although it only succeeds in giving the $L^{1}$ condition for $q_{x}(0, t)$ and for $t q_{x}(0, t)$ (which of course is enough for our purposes). In Sections 3 (after Theorem 3.1 and the resulting (3.25) ), and 4. we essentially imposed the extra conditions that the limits of certain derivatives exist as $t \rightarrow \infty$ (and thus, they are 0 , due for example to the fact that $q(x, t) \rightarrow 0$ as $t \rightarrow \infty$ uniformly for any $x$, or the $L^{2}(0, t)$ boundedness as $\left.t \rightarrow \infty\right)$. Thus, we were able to prove stronger decay estimates for $q_{x}(0, t)$, which in turn led also to the $L^{1}$ condition.

\section{REFERENCES}

[1] J. BARAB, Nonexistence of asymptotically free solutions for a Nonlinear Schrödinger equation, J. Math. Phys., 25 (1984), pp. 3270-3273.

[2] Jerry L. Bona, S. M. Sun And Bing-Yu Zhang, A non-homogeneous boundary-value problem for the Korteweg-de Vries equation in a quarter plane, Trans. Amer. Math. Soc. 354 (2002), pp. 427-490.

[3] J. Bourgain, Fourier transform restriction phenomena for certain lattice subsets and applications to nonlinear evolution equations, GAFA, 3 (1993), pp. 107-156.

[4] R. Carrol, Q. Bu, Solution of the forced Nonlinear Schrödinger (NLS) equation using PDE techniques, Applic. Anal., 41 (1991), pp. 33-51.

[5] J.E. Colliander, C.E. Kenig, The Generalized Kortewegde Vries Equation on the Half Line, CommPDE, 27 (2002), pp. 2187-2266.

[6] P. DeIfT, Orthogonal Polynomials and Random Matrices, AMS 2000.

[7] P. Deift, X.Zhou, Long-time asymptotics for solutions of the NLS equation with initial data in a weighted Sobolev space, Communications on Pure and Applied Mathematics, v.56 (2003), pp. 1029-1077.

[8] P. Deift, S.Venakides, X.Zhou, An extension of the steepest descent method for Riemann-Hilbert problems: The small dispersion limit of the Korteweg-de Vries (KdV)equation, Proc Natl Acad Sci U S A (1998), pp. 450454.

[9] P. Deift, X.Zhou, A Steepest Descent Method for Oscillatory Riemann-Hilbert Problems. Asymptotics for the MKdV Equation, Annals of Mathematics, Second Series, Vol. 137, No. 2 (1993), pp. 295-368

[10] A.S. FokAs, A Unified Transform Method for Solving Linear and Certain Nonlinear PDEs, Proc. R. Soc. Lond. A 453 (1997), pp. 1411-1443.

[11] A.S. FokAs, On the Integrability of Linear and Nonlinear PDEs, J. Math. Phys. 41 (2000), pp. $4188-4237$.

[12] A.S. FokAs, Integrable Nonlinear Evolution Equations on the Half-Line, Comm. Math. Phys. 230 (2002), pp. 139.

[13] A.S. FokAs, A Unified Approach to Boundary Value Problems, CBMS-NSF regional conference series in applied mathematics, SIAM 78, 2008.

[14] A.S. FokAs, And A. R. Its, The Nonlinear Schrodinger Equation on the Interval, J. Phys. A: Math. Gen. 37 (2004), pp. 6091-6114.

[15] A. S. FokAs, A. R. Its And L.-Y. Sung, The nonlinear Schrödinger equation on the half-line, Nonlinearity, 18 (2005), pp. 1771-1822. 
[16] A. S. Fokas, S. Kamvissis, Zero-dispersion limit for integrable equations on the half-line with linearisable data, Abstr. Appl. Anal. Volume 2004, Number 5 (2004), pp. 361-370.

[17] A. Boutet de Monvel, A. S. Fokas, D. Shepelsky, , Analysis of the Global Relation for the Nonlinear Schrdinger Equation on the Half-line, Letters in Mathematical Physics Volume 65, Issue 3, (2003), pp. 199-212.

[18] C.S. Gardner, J.M. Greene, M.D. Kruskal, and R.M. Miura, Kortewegi96;de Vries equation and generalizations. VI. Methods for exact solution, Comm. Pure Appl. Math., XXVII (1974), pp. 97-133.

[19] J. Holmer, The Initial-Boundary Value Problem for the Kortewegde Vries Equation, CommPDE, 31 (2006), pp. 1151-1190.

[20] S. Kamvissis, Semiclassical Nonlinear Schrödinger on the Half Line, J. Math. Phys., 44 (2003), pp. $5849-5869$.

[21] S. Kamvissis, On the Long Time Behavior of the Doubly Infinite Toda Lattice under Initial Data Decaying at Infinity, Comm. Math. Phys. 153 (1993) pp. 479-519.

[22] S. Kamvissis, K. Mclaughlin, P. Miller, Semiclassical Soliton Ensembles for the Focusing Nonlinear Schrödinger Equation, Annals of Mathematics Study v.154, Princeton 2003.

[23] S. Kamvissis, G. Teschl, Stability of periodic soliton equations under short range perturbations, Physics Letters A Volume 364, Issue 6 (2007), pp. 480-483.

[24] S. Kamvissis, G. Teschl, Long-time asymptotics of the periodic Toda lattice under short-range perturbations, J.Math.Phys. v.53 (2012).

[25] C.E. Kenig, G. Ponce, L Vega, Wellposedness and Scattering Results for the Generalized Kortewegde Vries Equation via the Contraction Principle, Comm. Pure Appl. Math., 46 (1993), pp. 527-620.

[26] C.E. Kenig, G. Ponce, L VegA, A bilinear estimate with applications to the KdV equation, JAMS, 9 (1996), pp. 573-603.

[27] D. Shepelsky, Personal communication.

[28] V.E. Zakharov, A.B. Shabat, Exact Theory of two-dimensional and one-dimensional self-modulation of waves in nonlinear media, Zh. Eksp. Teor. Fiz. 61 (1971), pp. 118-134. 